\title{
Detection of Characteristic Waves of Sleep EEG by Neural Network Analysis
}

\author{
Takamasa Shimada*, Tsuyoshi Shiina, Member, IEEE, and Yoichi Saito
}

\begin{abstract}
In psychiatry, the sleep stage is one of the most important evidence for diagnosing mental disease. However, doctors require much labor and skill for diagnosis, so a quantitative and objective method is required for more accurate diagnosis since it depends on the doctor's experience. For this reason, an automatic diagnosis system must be developed. In this paper, we propose a new type of neural network (NN) model referred to as a sleep electroencephalogram (EEG) recognition neural network (SRNN) which enables us to detect several kinds of important characteristic waves in sleep EEG which are necessary for diagnosing sleep stages. Experimental results indicate that the proposed NN model was much more capable than other conventional methods for detecting characteristic waves.
\end{abstract}

Index Terms-Charachteristic wave, EEG, neural network, sleep stage.

\section{ITRODUCTION}

I N PSYCHIATRY, sleep staging is one of the most important means for diagnosis. The sleep staging of electroencephalogram (EEG), however, is liable to be subjective since it depends on the doctor's skill and requires much labor. An automatic diagnosis system must, therefore, be developed to reduce doctor's labor and realize quantitative diagnosis of sleep EEG.

For sleep staging by EEG analysis, it is especially important to detect the characteristic waves from EEG. Most conventional methods of diagnosing the sleep stage, however, use long-term spectrum analysis [5], [6]. Such analysis is unable to detect transient and isolated characteristic waves such a hump wave from sleep EEG accurately. As a result, it is not possible to precisely diagnose the sleep stage based on characteristic waves as doctors do, though a roughly diagnosis is possible.

Moreover, some methods are based on a kind of template matching. This makes it difficult to cope with the large variation of EEG, such as fluctuations of the frequency pattern and the differences between individuals. The method of detecting the characteristic waves in EEG must, therefore, be able to recognize the time transient of the frequency pattern and be robust to variation of patterns.

Manuscript received July 3, 1998; revised September 2, 1999. Asterisk indicates corresponding author.

*T. Shimada is with the Applied Superconductivity Research Laboratory, Tokyo Denki University 2-1200 Muzai-Gakuendai, Inzai-shi, Chiba 270-1382, Japan (e-mail: shimada@asrl.dendai.ac.jp).

T. Shiina is with the Institute of Information Sciences and Electronics, University of Tsukuba 1-1-1 Tennoudai, Tsukuba-City, Ibaraki 305-8573, Japan.

Y. Saito is with the Research Institute for EEG Analysis 1-9-1-101 Nakasato, Kitaku, Tokyo 114-0015, Japan.

Publisher Item Identifier S 0018-9294(00)01774-2.
Recently, neural networks (NN's) have been applied to various kinds of problems in many fields due to their ability to analyze complicated systems without accurate modeling in advance [4]. In this paper, we propose a new type of $\mathrm{NN}$ model to detect several kinds of important characteristic waves in sleep EEG that are needed to diagnose sleep stages (e.g., sleep spindle, hump, and slow wave). We also compare our proposed method with other methods (two types of conventional NN models and the likelihood ratio method). Consequently, we show that our system can attain higher performance in detecting characteristic waves than these other methods.

\section{Detection Method by Neural Networks}

\section{A. SRNN Architecture}

Some characteristic waves in sleep EEG which are clues to diagnosis of sleep stage are short-term and appear sporadically in the background waves. A method for detecting them must, therefore, be able to take a large view of the EEG and recognize whether the characteristic waves are isolated or not. However, most conventional techniques for diagnosing the sleep stage are based on long-term spectral analysis and consequently not suited for detecting such transient and isolated characteristic waves.

We propose a new type NN model called SRNN for detecting transient characteristic waves in EEG based on the recognition of the two-dimensional (2-D) time-frequency pattern of characteristic waves. SRNN's architecture recognizes the sporadic properties of transient waves and performs shift-invariant processing. In other words, it detects transient waves regardless of where they appear within the input data window as shown in Fig. 1.

The time-delay neural network (TDNN) proposed by Waibel et al. [3] to detect the phoneme is a well-known NN, which has the shift-invariant function. It was shown that TDNN could be applicable if the position of the wavelet on the time axis could be roughly estimated. In other words, the deviation in location had to be small compared with the wavelet duration just like in detection of phoneme. However, for EEG, a shift larger than the wavelet duration must be compensated since we are unable to predict where characteristic waves appear within the input data window in advance.

To recognize the time-shifted pattern, SRNN has the following structure. First, time sequences of EEG data are transformed to a 2-D time-frequency data matrix where the ordinate is frequency and the abscissa is time. 


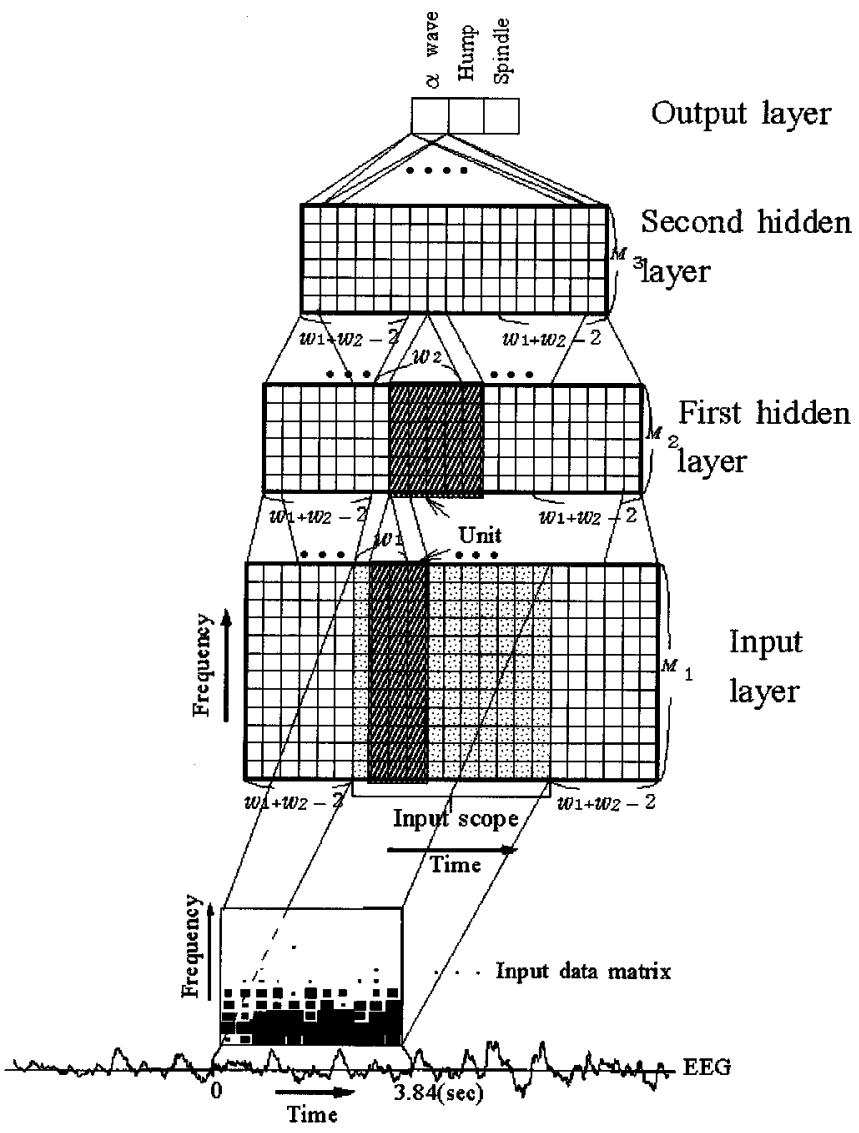

Fig. 1. SRNN architecture. This architecture recognizes the sporadic properties of transient waves and performs shift-invariant processing.

The reason why we applied the wide 2-D time-frequency matrix for input data instead of sliding the short window is that EEG includes some sporadic characteristic waves which are similar to background waves in short term spectrum. Therefore discrimination of the sporadic waves from background waves requires comparing the duration of spectrum pattern in the wide time window. In addition, the processing with the sliding window of the width corresponding to the detected waves is considerably time consuming, as a result, it is not proper especially for sleep EEG analysis due to its large amount of data (about 6-8 h).

Each element of the input data matrix is presented to the corresponding cell of the input layer. All input layer cells are covered with twenty-one unit. Each unit is composed of three successive column vectors of input layer cell and is arrayed to overlap the adjacent one by two column vectors (see Fig. 1). (Each unit is connected with the column vector of the first hidden layer. The values of the connection weights are made equal for every unit to attain the shift-invariant detection of transient waves.)

Five successive column vectors of the first hidden layer similarly compose units and are connected with a column of the second hidden layer in order to integrate the information on a lower layer over a large time range. The pattern of the connection weight is the same for every unit. All cells of the second hidden layer are connected with all three cells of the output layer, though the weights of connection are restricted. In other words, the pattern of weights connected with a neuron of output layer is made the same for every column of the second hidden layer.

SRNN is similar to TDNN with regard to the connection from the input layer to the second hidden layer. In TDNN, however, each row vector of the second hidden layer is connected with a single cell of the output layer, while in SRNN all cells of the two layers are connected. Another difference between SRNN and TDNN is that SRNN has an expanded area from the input layer to the second hidden layer in order to prevent sensitivity to the input data from becoming less at the edge of both sides than the center of the input layer because of lower density of connection at the edge. Let us consider architecture without the expanded region. If a unit is composed of $w_{1}$ columns in the input layer, then the data at the edge column of the first unit is transferred to the first hidden layer only once and that of second column from the edge is transferred twice. The number of connections between columns of the input layer and the upper layer increases to $w_{1}$. Similar nonuniformity of connection density occurs for connections between the first and the second hidden layers. To make the connection density uniform, each layer from the input layer to the second hidden layer is expanded along time axis as shown in Fig. 1. For example, if the input layer and the first hidden layer are composed of $w_{1}$ and $w_{2}$ column vectors, $w_{1}+w_{2}-2$ columns must be added to both sides of the input layer and the two hidden layers. Moreover, zero values are embedded at expanded area of input layer where is covered by first hidden layer units and processed as nonsignal area. Even if the first hidden layer unit covers only expanded area of input layer, the output value of neuron is not zero because of the threshold value of neuron. By this method, the data at the edge column is transferred to both expanded and nonexpanded area and it solves the nonuniformity problem of connection density between edge and center.

In the processing described later, as we showed in Fig. 1, the input layer is composed of a 12-row, 23-column matrix. A unit of the input layer consists of three columns. The first hidden layer is composed of six rows and 21 columns, and its unit consists of five columns. The second hidden layer is composed of six rows and 17 columns. The back-propagation algorithm was applied in the learning procedure.

\section{B. Detection with NN}

As described earlier, SRNN must recognize a 2-D time-frequency pattern of characteristic waves. Input data then forms a 2-D matrix; the horizontal axis indicates time, and vertical axis, frequency.

The training or tutorial data for the learning process is generated as follows. First, the EEG data is sampled (at the rate of $200 \mathrm{~Hz}$ ) with a 0.64-s (128-point) hamming window. Its logarithmic power spectrum coefficients are calculated by fast Fourier transform (FFT) and 12 coefficients of lower frequency are normalized by (1) and (2), and used for input data. Generally, most of power spectrum of characteristic waves detected later (spindle, hump, and alpha wave) are included in this spectrum range (from zero to $18.2 \mathrm{~Hz}$ ). And we applied the 
normalizing with range of $\pm 3 \sigma$ which covers $90 \%$ of amplitude of Gaussian distribution to attain the stable detection rate by rejecting noise with large magnitude which is often included in EEG

$$
\begin{aligned}
& f_{k}=k \Delta f\left(k=0,1,2, \cdots, 11 \quad \Delta f=\frac{200}{128} \cong 1.56 \mathrm{~Hz}\right) \\
& P^{\prime}\left(f_{k}\right)= \begin{cases}1 & \left(m+3 \sigma<P\left(f_{k}\right)\right) \\
\frac{P\left(f_{k}\right)-m}{3 \sigma} & \left(m \leq P\left(f_{k}\right) \leq m+3 \sigma\right) . \\
0 & \left(P\left(f_{k}\right)<m\right)\end{cases}
\end{aligned}
$$

Where $P\left(f_{k}\right)$ is the logarithmic power spectrum coefficient, and $m$ and $\sigma$ are the mean and standard deviation of all logarithmic spectrum data over whole training data, respectively.

One data matrix consists of 11 logarithmic spectrum data sets so that the time duration of one matrix corresponds to $3.84 \mathrm{~s}$ and the frequency ranges from $0 \mathrm{~Hz}$ to $17.2 \mathrm{~Hz}$. The EEG data is processed by shifting the input data window or the input layer by a half size of the window, i.e., $1.29 \mathrm{~s}$.

As a similar work, Eberhard et al. detected the epileptic spike detection by using raw EEG data as an input of the network [8]. The epileptic spike is characterized as much narrower waveform than background wave and is easy to detect base on the time structure of the wave. However, characteristic wave of sleep EEG, especially humps, has more various waveform with longer duration than the epileptic spike. Therefore, in order to extract the common feature of the characteristic waves independent of the variation, spectral parameter is more desirable than time sequence pattern, which is too sensitive to the detail of the waveform.

We used the following five types of characteristic waves;
1) spindle;
2) hump;
3) alpha wave;
4) hump train (continuous hump);
5) background wave.

Fig. 2 [mibel11] shows the 2-D time-frequency pattern of five characteristic waves. It should be noted that the hump train does not exist in the real EEG and was produced to make SRNN learn the sporadic properties of humps. The short-term spectral pattern of background waves resembles that of humps so that sporadic properties of humps are used to discriminate them from background. That is why the hump trains were generated by combining many humps. Using these training data, all connection weights are adjusted so as to decrease the error by the back-propagation learning procedure based on the gradient decent method (3) and (4) [1], [2]

$$
\begin{gathered}
E=\frac{1}{2} \sum_{j} \sum_{c}\left(y_{j, c}-\bar{y}_{j, c}\right)^{1 / 2} \\
\Delta \omega_{p, q}=\varepsilon \frac{\partial E}{\partial \omega_{p, q}}(\varepsilon>0)
\end{gathered}
$$

where $y_{j, c}$ and $\bar{y}_{j, c}$ represent the output and desired output of the $j$ th output neuron for the input pattern $c$. Parameter $\varepsilon$ represents the velocity coefficient of convergence of NN learning.
The procedure performs two passes through the network. One is the forward pass, and another is backward pass. In forward pass, when an input pattern is applied to input layer, the output values are calculated through the connection weights. In the backward pass, the output values are compared with the desired output values and its error is calculated [(3)]. This error is back propagated through the network and the connection weight is changed by gradient descent of the mean squared error as a function of weights (between neuron $p$ and $q$ changed by $\Delta \omega_{p, q}$ ).

The NN is trained so that the corresponding output neuron fires when a spindle, hump or alpha wave is applied to the input layer; output neurons do not fire when a hump-train or background wave is inputted.

In this paper, data for three persons (A, B, and C) was analyzed. The data includes wake stage, stage 1 , and stage 2 . And its total time is about $10 \mathrm{~min}$. The training data was developed based on subject A's data, and it includes ten spindles, 134 humps, 14 alpha waves, 49 hump trains, and 66 background waves.

Training was repeated many times for all the training data until the network weights converge so as to produce the desired output. Fig. 3 shows the time transient curve of the squared error during SRNN's training. The error converged after 24000 iterations, taking $5 \mathrm{~h}$ on personal computer. We should note, however, that this amount of computation is required only for training, not for recognition. Once the training procedure is completed, the recognition procedure can be executed in a few minutes for $1 \mathrm{~h}$ of EEG data. It is also clear that we can implement the training procedure much faster by using a high-performance workstation.

The examined EEG data was similarly sampled using the window and transformed to the input data matrix. The input data windows are shifted, and the characteristic waves corresponding to the neuron are regarded to be detected at each position where the fire level of an output neuron exceeds a threshold.

\section{Comparison of Performances with Other Methods}

The performance of SRNN in detecting characteristic waves of EEG was evaluated by comparison with the other methods [i.e., TDNN, the all connecting neural network (ACNN), and the likelihood ratio method based on AR coefficient (LRM)].

TDNN and ACNN were selected as the methods using NN's. As mentioned above, TDNN has been developed to detect phoneme, and its architecture is similar to SRNN. As shown in Fig. 4, the TDNN used here consists of an input layer with 12 rows and 11 columns, the first hidden layer with 17 rows and nine columns, the second hidden layer with three rows and five columns and the output layer with three neurons. Three columns of the input layer are connected with a column of the first hidden layer, and five columns of the first hidden layer are connected with a column of the second hidden layer. Each row is connected with one of the output neurons.

The ACNN has the most general architecture of back-propagation-based NN's and its connection weights are not constrained like SRNN or TDNN as shown in Fig. 5. It has six cells in the first hidden layer and ten cells in the second hidden layer. The numbers of neuron cells of these NN's are adjusted to be approximately equal. 
$(\mathrm{Hz})$

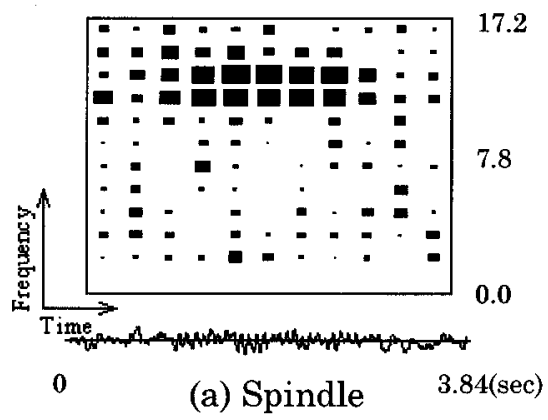

$(\mathrm{Hz})$

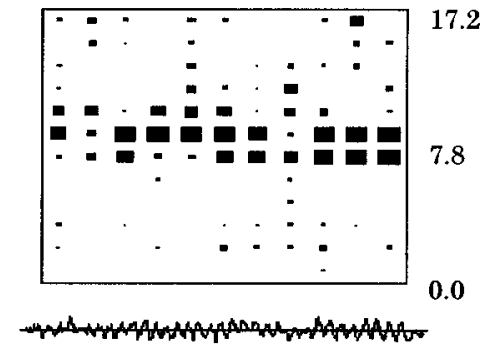

0

(c) Alpha wave $3.84(\mathrm{sec})$

$(\mathrm{Hz})$

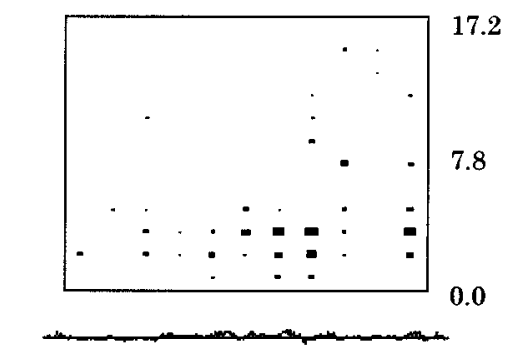

0 (e) Background wave ${ }^{3.84(\mathrm{sec})}$
$(\mathrm{Hz})$

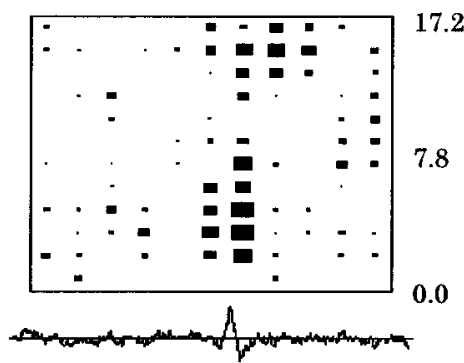

(b) Hump 3.84(sec)

$(\mathrm{Hz})$

17.2

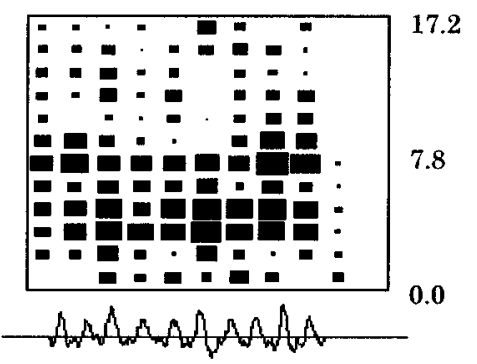

0 (d) Hump-train $\quad 3.84(\mathrm{sec})$

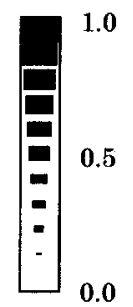

Scale

Fig. 2. Waveform and input data matrix of characteristic waves used. The EEG data is sampled (at the rate of $200 \mathrm{~Hz}$ ) with a 0.64-s (128-point) hamming window. Its logarithmic power spectrum coefficients are calculated by FFT and 12 coefficients of lower frequency are normalized by (1) and (2).

The LRM was selected as a typical template matching method. The template for characteristic waves was obtained by averaging each kind of wave in the training data. The likelihood ratio $D$ between the template signal and the examined EEG data defined by (5) was calculated

$$
D\left(P_{x} \mid P_{y}\right)=\log \left(a_{y} c_{x}^{T} a_{y} / a_{x} c_{x}^{T} a_{x}\right)+\log \left(a_{x} c_{y}^{T} a_{x} / a_{y} c_{y}^{T} a_{y}\right)
$$

where $P_{x}$ represents the optimum autoregressive (AR) model; $a_{x}$, the linear prediction coefficients; and $c_{x}$, the auto correlation vector of the template signal. $P_{y}, a_{y}$, and $c_{y}$ represent those parameters for the examined EEG data.

$D$ is equal to zero when the examined data coincides exactly with the template. Therefore, for a given template, the examined data is detected as the characteristic wave corresponding to the template when $D$ is less than a threshold value.

Three templates for the following characteristic waves were adopted:

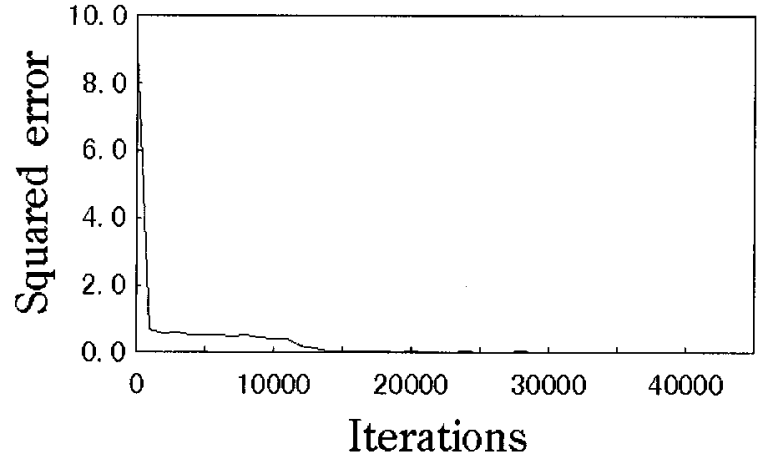

Fig. 3. SRNN output error versus number of learning iterations.

1) spindle;

2) hump;

3) alpha wave.

The duration of the examined EEG data is set at $3.84 \mathrm{~s}$ (768 points) which is equal to the case of NN's for the comparison. 


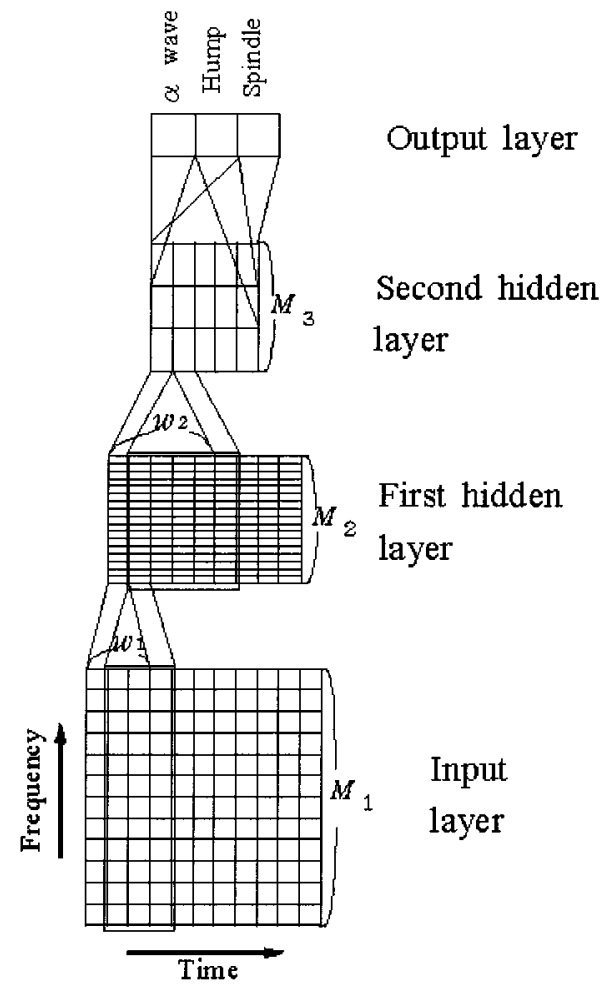

Fig. 4. TDNN architecture that has been developed to detect phoneme.

The order for AR coefficients was chosen as eight in the experiment since results with orders other than eight made no difference.

\section{RESULTS}

We applied SRNN, ACNN, TDNN, and the likelihood ratio method to the examined EEG data of three subjects, A, B, and $\mathrm{C}$ as described in Table I and detected three types of characteristic waves (spindle, hump, and alpha wave). Data points for the subjects are shown in Table II (sample rate is $200 \mathrm{~Hz}$ ).

The averaged detection rates for open experiments and closed experiments are shown in Fig. 6(a) and (b). An open experiment means that the learning data does not include the examined data, a closed experiment does. The detection rate is defined as shown in (6) found at the bottom of the page.

Next, we tried to test the significance of the difference between these results. We applied the null hypothesis that the averaged detection rates of SRNN, TDNN, ACNN, and likelihood ratio method make no difference, and tested with a significance level of 5\%. Table III shows the results of the test. If the null hypothesis was rejected, an $\times$ was entered in the corresponding position. Fig. 6 and Table III indicate that the SRNN detection rate is significantly higher than that of other methods for most cases.

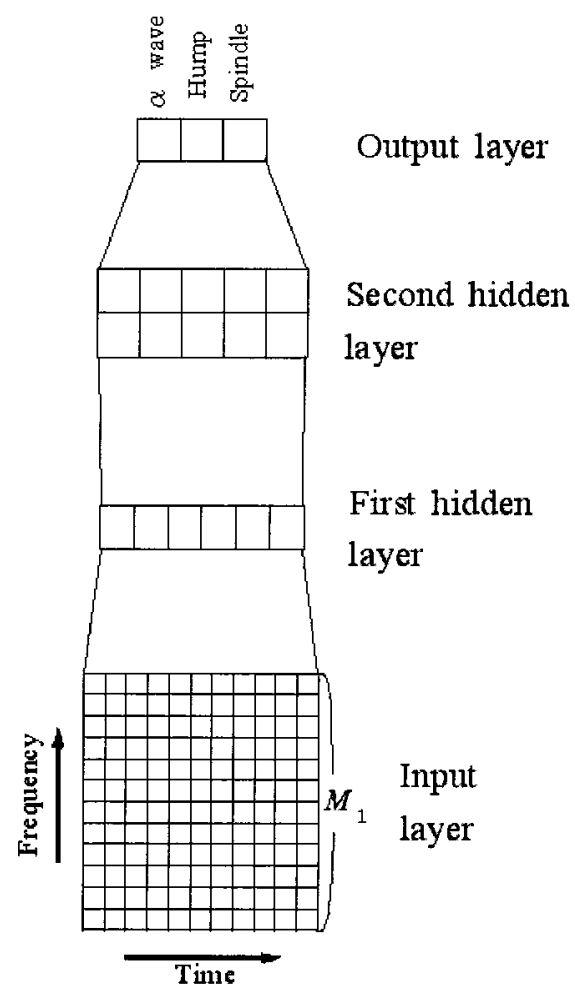

Fig. 5. ACNN architecture. This architecture is the most general of back-propagation-based NN's and its connection weights are not constrained like SRNN or TDNN.

TABLE I

Conditions of SubJects PRoviding EEG DATA USED IN EXPERIMENTS

\begin{tabular}{|c|c|c|c|}
\hline Subject & $\begin{array}{c}\text { Age } \\
\text { (years) }\end{array}$ & Gender & $\begin{array}{l}\text { Doctor's } \\
\text { opinion }\end{array}$ \\
\hline A & 35 & Female & Normal \\
\hline B & 58 & Female & $\begin{array}{l}\text { Other function } \\
\text { psychoses }\end{array}$ \\
\hline C & 21 & Female & Normal \\
\hline
\end{tabular}

TABLE II

DATA POINTS FOR THE SUBJECTS

\begin{tabular}{c|c}
\hline Subject & Data Points \\
\hline A & 69,632 \\
B & 65,408 \\
C & 77,824 \\
\hline
\end{tabular}

\section{INTEGRATION OF MULTICHANNEL INFORMATION}

Medical doctors usually use multichannel EEG sequences measured at different portions on the scalp to improve the detection rate by integrating global information on characteristic waves. Therefore, we tried to use the integrated multichannel information to detect characteristic waves.

$$
\text { Detection rate }=\frac{\text { The number of correctly detected matrices }}{\text { The number of matrices which includes the objective characteristic wave }} \times 100[\%]
$$




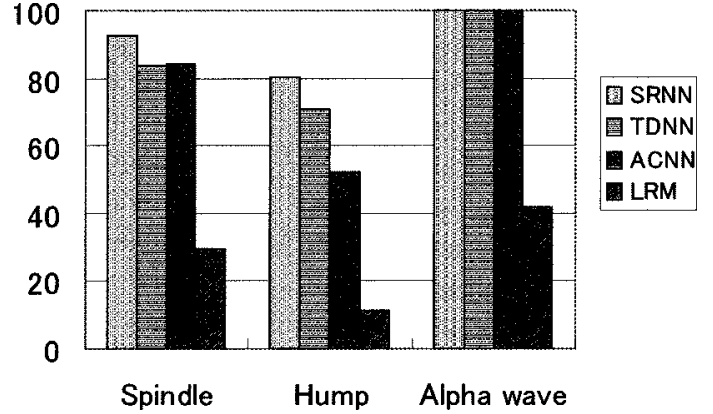

(a)

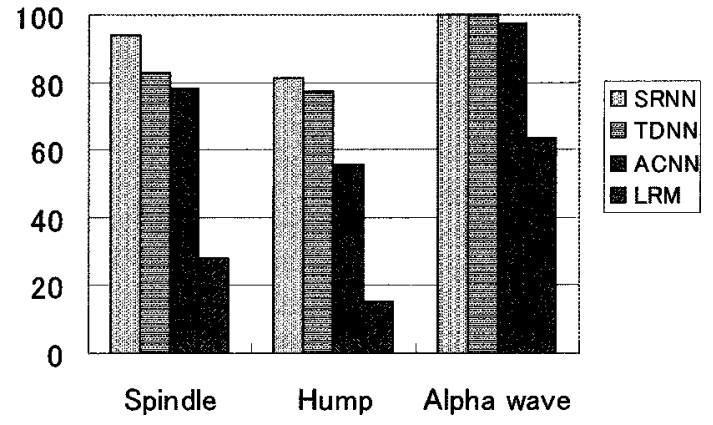

(b)

Fig. 6. Comparison of detection rate of several methods with (a) opened and (b) closed experiments.

Output neurons often misfire due to low amplitude of characteristic waves, overlapping of large background waves, and so on. The integration of the multichannel information should compensate for the output of misfired channels.

In our experiment, we used eight channels (Fp1, Fp2, C3, C4, $\mathrm{P} 3, \mathrm{P} 4, \mathrm{O} 1$, and $\mathrm{O} 2$ ) based on a ten-twenty electrode system.

To integrate multichannel information, we employed a simple summation of the output signal at each channel by SRNN as described by (7). This means OR-like operation. Next, the summation was used to obtain the final detection

$$
\begin{aligned}
\bar{O}_{n} & =\sum_{k=1}^{s} O_{k} \\
\eta_{n} & =F\left[O_{n} ; \lambda\right]
\end{aligned}
$$

where $O_{k}$ is the output of each channel, $F$ is a sigmoid function and $\lambda$ is the threshold. Fig. 7(a) shows the results of detecting humps by integrating multichannel data compared with single-channel processing. It can be seen that in spite of the very simple processing or summation of output at each channel, the result designated by "all" in Fig. 7(a) realized the maximum detection rate. This apparently indicates that multichannel analysis improves the detection rate by compensating for the channel with misfire.

In contrast, the result of multichannel processing similarly applied to LRM did not achieve the highest performance as shown in Fig. 7(b).

We may explain the difference of performance between SRNN and LRM by considering whether the decision process is nonlinear or not. Table IV shows the ratio of the mean firing level of output neurons for humps compared to that for other
TABLE III

TeSt of Significance of Difference in Detection Rate

(a) Subject A closed

\begin{tabular}{c|c|c|c} 
& \multicolumn{4}{|c|}{ (a) Subject A closed } & \\
\hline & Spindle & Hump & $\alpha$ wave \\
\hline TDNN & $\times$ & $\times$ & \\
\hline ACNN & $\times$ & $\times$ & \\
\hline LRM & $\times$ & $\times$ & $\times$ \\
\hline
\end{tabular}

(b) Subject B open

\begin{tabular}{c|c|c|c} 
& Spindle & Hump & $\alpha$ wave \\
\hline TDNN & $\times$ & $\times$ & \\
\hline ACNN & $\times$ & $\times$ & \\
\hline LRM & $\times$ & $\times$ & $\times$ \\
\hline
\end{tabular}

(c) Subject B closed

\begin{tabular}{c|c|c|c} 
& Spindle & Hump & $\alpha$ wave \\
\hline TDNN & $\times$ & & \\
\hline ACNN & $\times$ & $\times$ & $\times$ \\
\hline LRM & $\times$ & $\times$ & $\times$ \\
\hline
\end{tabular}

(d) Subject B open

\begin{tabular}{c|c|c|c} 
& Spindle & Hump & $\alpha$ wave \\
\hline TDNN & $\times$ & $\times$ & \\
\hline ACNN & $\times$ & $\times$ & \\
\hline LRM & $\times$ & $\times$ & $\times$ \\
\hline
\end{tabular}

(e) Subject $\mathrm{C}$ closed

\begin{tabular}{c|c|c|c}
\hline & Spindle & Hump & $\alpha$ wave \\
\hline TDNN & $\times$ & $\times$ & \\
\hline ACNN & $\times$ & $\times$ & \\
\hline LRM & $\times$ & $\times$ & $\times$ \\
\hline
\end{tabular}

(f) Subject $C$ open

\begin{tabular}{c|c|c|c} 
& Spindle & Hump & $\alpha$ wave \\
\hline TDNN & $\times$ & $\times$ & \\
\hline ACNN & $\times$ & $\times$ & \\
\hline LRM & $\times$ & $\times$ & $\times$ \\
\hline
\end{tabular}

patterns. It can be seen that the SRNN firing level is much higher than that of LRM since the nonlinear SRNN system outputs a sufficient level for the definite existence of the target wavelet and suppresses the output for ambiguous cases. As a result, a channel with an ambiguous fire (fired value is near zero) affects the integrated output less. The LRM firing level is produced by significantly linear system. Consequently, the output level can not be neglected for an ambiguous fire, and influences the integrated values.

\section{DISCUSSION}

The above results indicate that all three NN's attained high detection rates for alpha waves, for humps, however, the SRNN result was better than others. It is reasonable to believe that these 


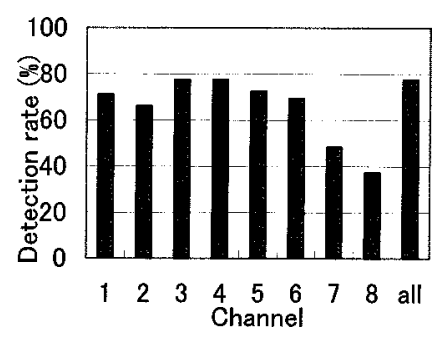

(a)

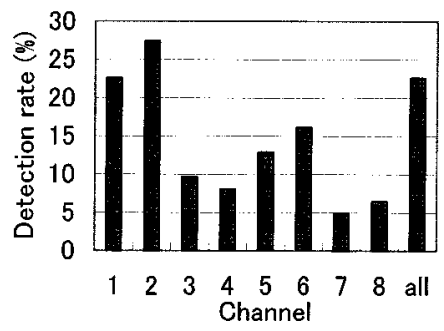

(b)

Fig. 7. Comparison of the effect of multichannel processing on detecting humps between (a) SRNN detection rate and (b) LRM detection rate.

TABLE IV

RATIO OF MEAN FIRING LEVEL FOR HUMPS AND THAT FOR OTHER WAVE PATTERNS

\begin{tabular}{c|c|c}
\hline & SRNN & LRM \\
\hline Ratio & 35.07 & 6.72 \\
\hline
\end{tabular}

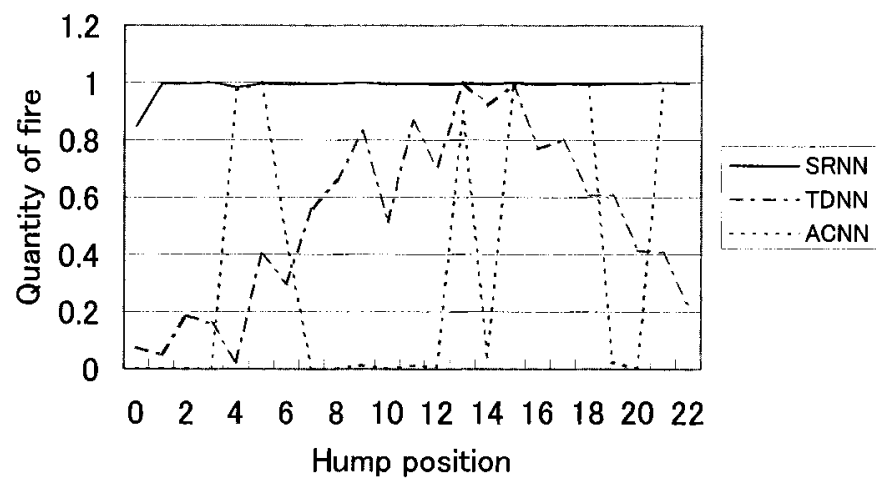

Fig. 8. Stability of output neuron's quantity of fire of SRNN, TDNN, and ACNN. These results indicate that the fire level in SRNN is more stable than that in TDNN or ACNN.

results were due to the difference in abilities to detect shorter wavelets compared with the size of the input data window.

To detect short-duration wavelets like humps, NN's require two kinds of ability. One is the time-shift-invariant detection because the location characteristic waves appear within the input matrix is not known in advance. The other is the ability to recognize the sporadic properties of characteristic waves since the local spectrum properties are not enough to distinguish the wavelets from a background wave whose local spectrum pattern is similar.

SRNN also attained high performance for long-duration wave such as spindles, but the detection rates of LRM are much lower.

In the following, we investigate these aspects in detail.

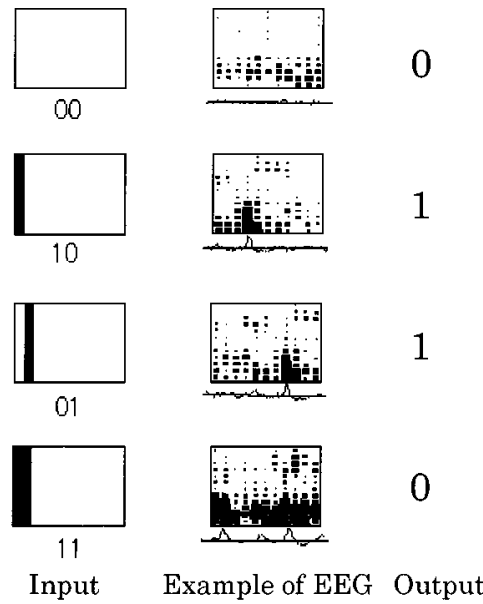

Fig. 9. Input patterns for XOR-like operation.

\section{A. Shift-Invariant Function}

To detect short-duration characteristic waves, an NN must have a time-shift-invariant function, i.e., the ability to detect wavelets uniformly regardless of their appearance location within the input window.

For TDNN, although it has a time-delay structure similar to that of SRNN, the number of operations at the edge of each layer is smaller than at the center, so detection sensitivity is not uniform.

ACNN does not have a time-delay structure like the other two NN's. Therefore, to learn the properties of characteristic waves uniformly, the training data must be uniformly presented to the input layer, but this is not easy in practice.

We next examined whether SRNN, TDNN, and ACNN can learn EEG patterns uniformly, regardless of the location of the characteristic waves within the input layer, by detecting the hump while shifting it gradually (23 steps) from left to right in the input data window. Signals other than humps within the window were filled with the background waves observed typically at sleep stage II. As we show in Fig. 8, the experiment results indicate that the fire level in SRNN is more stable than that in TDNN or ACNN. The TDNN firing level has a peak at the center of the input data window and becomes lower at the edge. The ACNN firing level has a large variation all over the input data window. In contrast, the SRNN firing level is almost constant regardless of the hump's position, which means that SRNN can recognize short characteristic waves uniformly, and can, consequently, achieve their time-shift-invariant detection within the input data window.

\section{B. Recognition of the Isolation of Characteristic Waves}

Let us consider another reason why as shown in Fig. 6 the SRNN has a much better detection rate for hump waves than TDNN, even though both have a time-delay structure and both had sufficiently high detection rates for alpha waves. If we represent humps as " 1 " and background wave as " 0, " the ideal output must be as follows: when "1" is input to at least one unit of the input layer, the output must indicate the detection of humps. However, when "1" is input to some of adjacent units, the firing of output cells for humps must be suppressed because 


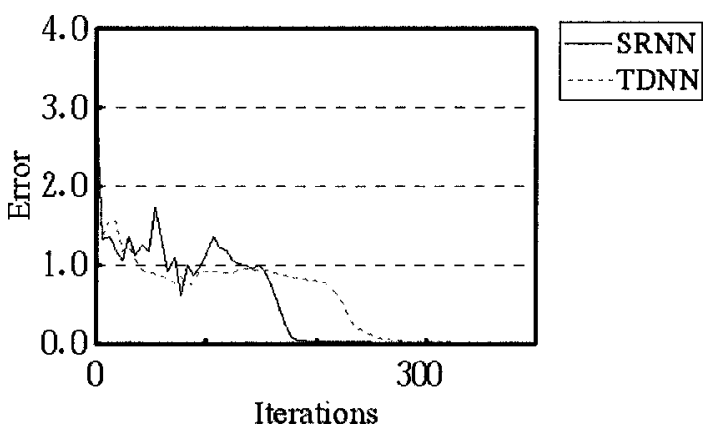

(a)

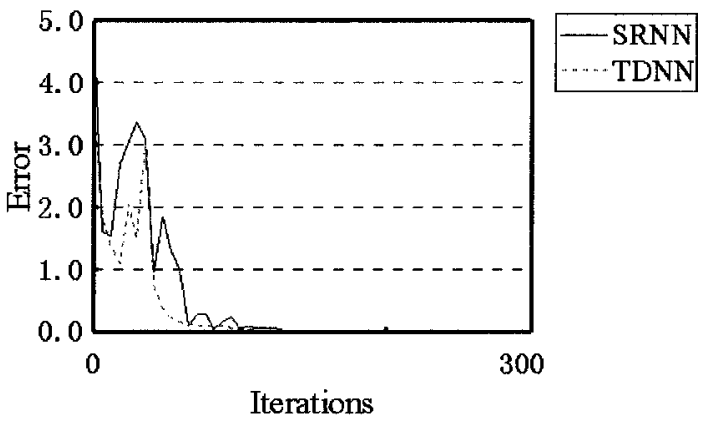

(b)

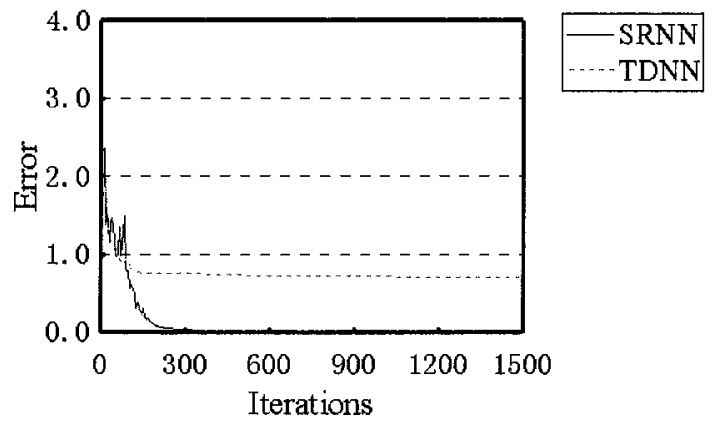

(c)

Fig. 10. Output error versus number of learning iterations for (a) AND, (b) OR, and (c) XOR operations by TDNN and SRNN. For XOR operations, SRNN converged after 400 iterations, but TDNN did not converge even after 1500 iterations as shown in (c).

humps are sporadic and not trained, though their spectral components are similar to those of other types of long-duration characteristic waves such as slow waves and background waves. One may then say that these processes for detecting isolated characteristic waves like humps correspond to an XOR-like operation. This hypothesis makes it possible to understand that the two NN's differ in capacity for XOR-like operation, which is necessary to recognize the sporadic properties or the isolation of humps.

NN's require three sufficiently connected layers to implement an XOR operation [4]. However, as mentioned in Section II-C, TDNN has a more constrained connection than SRNN between the second hidden layer and the output layer. SRNN has an all-connection structure with the same value along the horizontal direction. In contrast, the TDNN output is connected to a specific row of the second hidden layer. From this viewpoint, SRNN may be expected to be superior to TDNN in XOR operation.

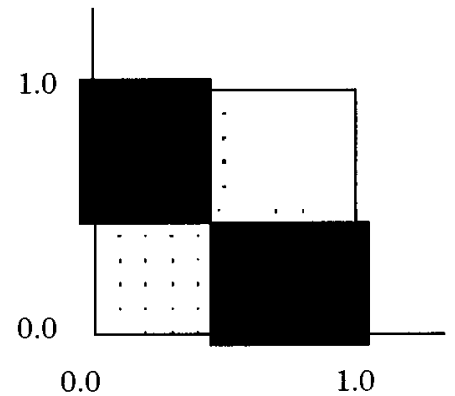

(a)

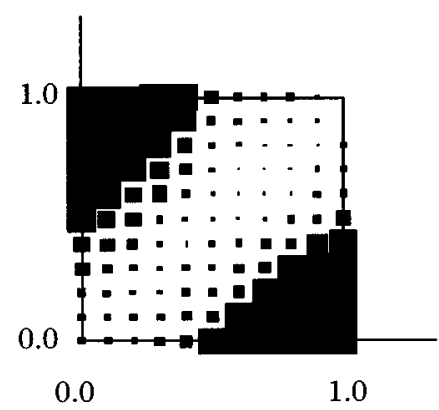

(b)

Fig. 11. XOR decision regions implemented by (a) SRNN and (b) TDNN. It can be seen that the XOR decision region of SRNN is correct while that of TDNN is not.

To confirm this, we used a simple model of input data in which a column vector $\mathbf{1}=(1,1 \cdots 1)^{T}$ is regarded as the spectral pattern of the hump and $\mathbf{0}=(0,0, \cdots, 0)^{T}$ is regarded as the spectral pattern of the background wave in the input data matrix. The symbol [0 0 ] in the upper left of Fig. 9 means that the input data matrix is filled with zeros, which correspond to the background waves. Consequently, the output should be "0" as shown on the right. In the second and third figures from the top, $\left[\begin{array}{ll}\mathbf{0} & \mathbf{1}\end{array}\right]$ and $\left[\begin{array}{ll}\mathbf{1} & \mathbf{0}\end{array}\right]$ mean that one of the two adjacent columns is $\mathbf{1}$, which corresponds to the input of the hump in each position as shown in the center. The output "1" is then expected. The bottom [ $\left.\begin{array}{ll}1 & 1\end{array}\right]$ corresponds to the hump train described in Section II. If the XOR-like operation is implemented correctly, the output neuron becomes "1" only when the input pattern is $\left[\begin{array}{ll}0 & 1\end{array}\right]$ or $\left[\begin{array}{ll}\mathbf{1} & \mathbf{0}\end{array}\right]$. In practice, these patterns were gradually shifted within the input matrix from left to right.

These input patterns were input as training data to both SRNN and TDNN to evaluate their performance for AND, OR, and XOR operations.

Fig. 10 shows the results. The abscissa is the number of learning iterations, and the ordinate is the squared error of output. As shown in Fig. 10(a) and Fig. 10(b), both SRNN and TDNN converged after 300 iterations for AND and OR operations. For XOR operations, SRNN converged after 400 iterations, but TDNN did not converge even after 1500 iterations as shown in Fig. 10(c). Fig. 11 shows the decision regions of XOR operations implemented by SRNN and TDNN. It can be seen that the XOR decision region of SRNN is correct while that of TDNN is not. This indicates that SRNN has much more capacity for XOR-like operation. 

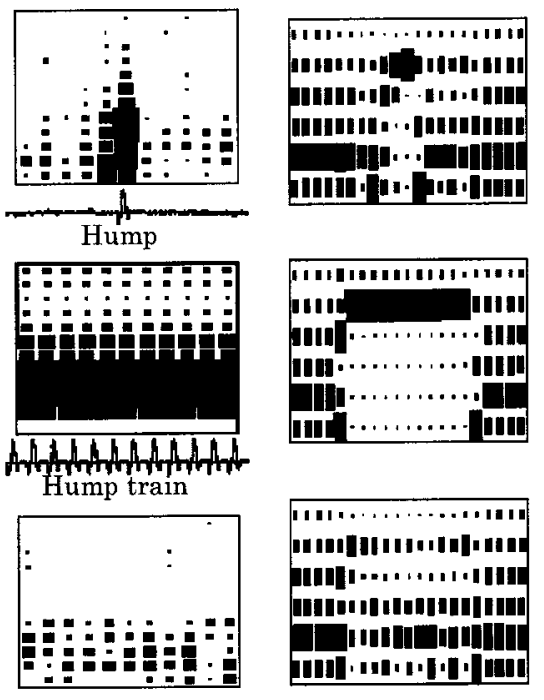

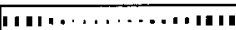
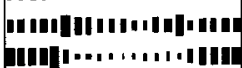

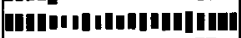

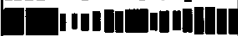

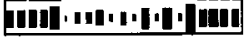

Background wave

(a) Input pattern

(b) First hidden layer

Fig. 12. Input, output, and firing patterns of each layer.

Finally, we examined the connection weights of SRNN in detail. Fig. 12 shows the pattern of the input data matrix, and the corresponding output and firing pattern of each layer for humps, hump train, and background wave.

It can be seen that neurons on the second row of the first hidden layer fire for both the hump and the hump train. This reveals that the second row of neurons corresponds to the spectrum band of the hump but is insufficient for recognizing the isolation of characteristic waves. As shown in Fig. 12(c), neurons on the top row of the second hidden layer fire for both the hump and the hump train, while neurons on the second row fire only for the hump train. We can see from these results that neurons on the top row of the second hidden layer perform an OR operation and those on the second row perform an AND operation on the spectrum pattern of humps appearing in the first hidden layer. Generally, XOR operation is defined as

$$
(\mathrm{A} X O R \mathrm{~B})=(\mathrm{A} \text { OR B })-(\mathrm{A} \text { AND B })
$$

According to (8), the connection weights between the OR output of the second hidden layer and the output layer should be positive for XOR operation and while that between the AND output and the output layer should be negative. Practical connection weights between the second hidden and the output layer for humps are shown in Fig. 13 where area of boxes is proportional to amplitude of connection weights and black boxes represent positive values and white boxes negative. These could ensure implementation of XOR-like operations in SRNN. Consequently, the output neurons of humps fire only when humps are input to the input layer, and SRNN could recognize these patterns correctly. The value of the top box in Fig. 13(b) is positive and corresponds to OR, while the second box is negative and corresponds to AND.
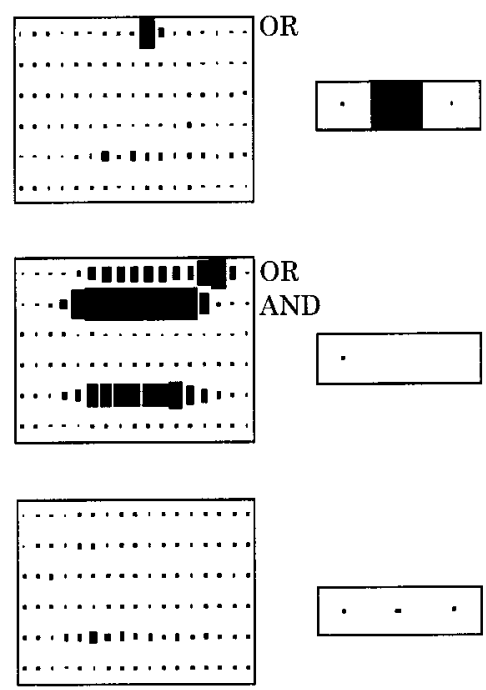

(c) Second hidden layer

(d) Corresponding output firing

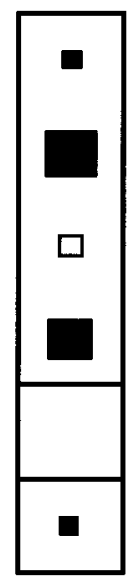

(a) Alpha wave

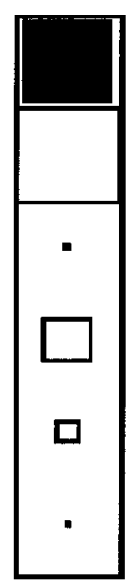

(b) Hump

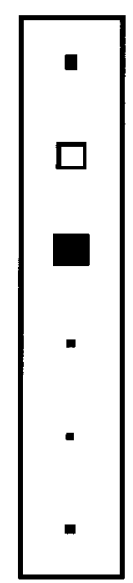

(c) Spindle
Fig. 13. Connection weights between second hidden layer and output layer; Area of boxes is proportional to amplitude of connection weights. Black and white boxes represent positive and negative values, respectively. These could ensure implementation of XOR-like operations in SRNN.

\section{Influence of the Characteristic Wave Duration}

Fig. 7 shows that the LRM detection rates are considerably lower than those of SRNN, even for spindle and alpha waves whose durations are longer than humps. One explanation for these results may be that the performance of LRM for detecting long-duration characteristic waves depends on how much the window is occupied by a part of the characteristic wave. We examined this point by using the spindle model (burst waves of $14 \mathrm{~Hz}$ having various time durations and embedded in random signals as background waves).

Fig. 14 shows the simulation results. The abscissa indicates the ratio, $\mu$, at which the window is occupied by the spindle, and the ordinate is the likelihood ratio. The top figure illustrates waveform within the input window and waveform in the right 


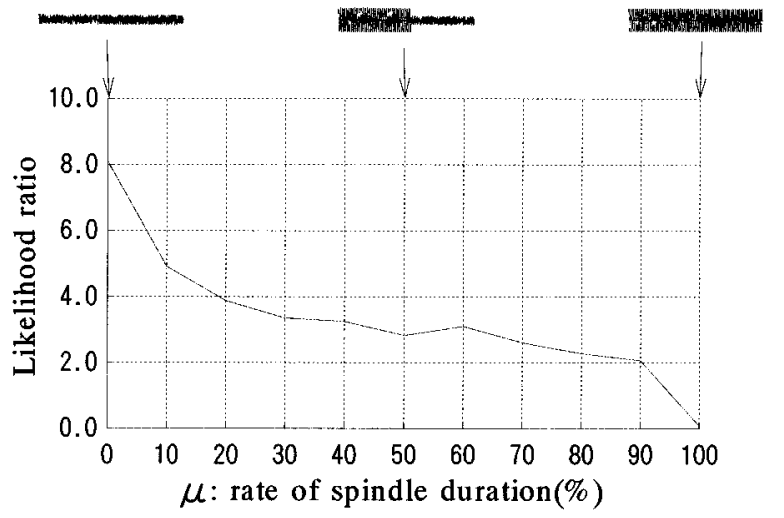

(a) Likelihood ratio versus rate of spindle duration to input window size

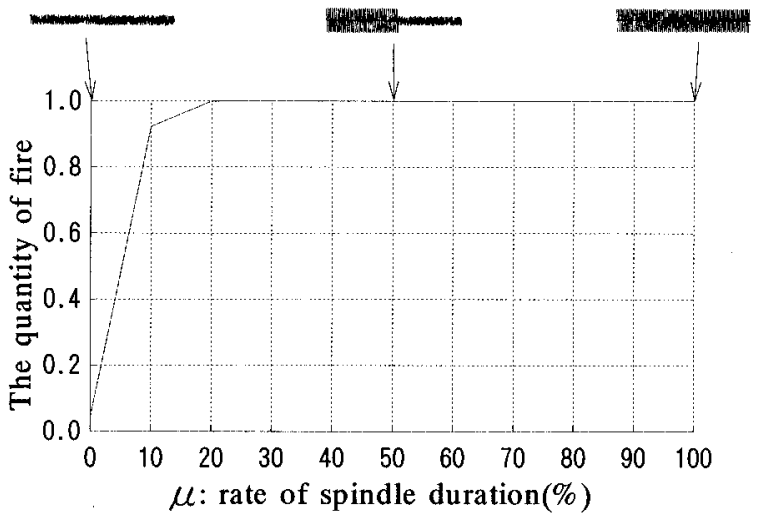

(b) SRNN output firing versus rate of spindle duration to input window size

Fig. 14. Comparison of influence of characteristic wave duration between LRM and SRNN. These results indicate that the SRNN output is almost independent of the ratio $\mu$ and produces a constant level except for the range of very small ratios.

side corresponds to a template of LRM. This figure shows that as $\mu$ becomes lower, the difference between the template and objective wave becomes larger; consequently, the likelihood becomes higher. This prevents LRM from identifying identical two waves with differing positions in the window.

In contrast, Fig. 14(b) indicates that the SRNN output is almost independent of the ratio and produces a constant level except for the range of very small ratios.

Generally, a template matching method like LRM is not suited for detecting patterns with large variance, and in the above case such a variance is caused by a time shift of the wavelet in the input matrix.

\section{CONCLUSION}

We proposed the SRNN for detecting the characteristic waves of sleep EEG and evaluated its performance by experiment.

Comparison of SRNN to three other methods, ACNN, TDNN, and LRM, revealed that a better detection rate was attained by using SRNN to detect characteristic waves regardless of the duration of wavelets. We also tried to use the multichannel information of the SRNN output, and showed that by integrating the output of each channel a higher detection rate was attained than by selecting a particular channel since the correct output compensates for the nondetected channels.

We confirmed that SRNN has a higher detection rate than other methods and investigated the reasons. We illustrated that SRNN could learn EEG patterns uniformly regardless of the location in the input layer by means of a time-delay structure in which the input layer and hidden layer are composed of many arrayed units and governed by the same connection weights. Another reason SRNN could learn EEG patterns is its capability to recognize properties of the isolation. This capability is implemented by an XOR-like operation. By examining the SRNN connection weights, we confirmed that the SRNN structure could execute XOR operations and, consequently, has sufficient capacity for recognizing the sporadic characteristics.

Finally, it was shown that SRNN was less influenced than LRM by the duration of the characteristic wave. As a result, higher detection rate was attained by SRNN even for long-duration characteristic waves.

For future study, the proper size of the NN, the input data normalizing method and so on must be investigated. Moreover, we are now trying to apply SRNN to classifying sleep stages based on EEG data.

\section{REFERENCES}

[1] D. E. Rumelhart, G. E. Hinton, and R. J. Williams, "Learning representations by back-propagation errors," Nature, vol. 323, pp. 533-536, Oct. 1986.

[2] — Learning Internal Representations By Error Propagation. Cambridge, MA: M.I.T. Press, 1986, ch. 8, pp. 318-362.

[3] A. Waibel, T. Hanazawa, G. Hinton, and K. Lang, "Phoneme recognition using time-delay neural networks," IEEE Trans. Acoust., Speech, Signal Processing, vol. 37, pp. 328-339, Mar. 1989.

[4] R. P. Lippmann, "An introduction to computing with neural nets," IEEE Acoust. Speech, Signal Processing Mag., pp. 4-22, Apr. 1987.

[5] J. C. Principe and J. R. Smith, "SAMICOS-A sleep analyzing microcomputer system," IEEE Trans. Biomed. Eng., vol. BME-33, pp. 935-941, Oct. 1986.

[6] J. C. Principe, S. K. Gala, and T. G. Chang, "Sleep staging automaton based on the theory of evidence," IEEE Trans. Biomed. Eng., vol. 36, pp. 503-509, May 1989.

[7] K. Fukushima, S. Miyake, and T. Ito, "Neocognitron: A neural network model for a mechanism of visual pattern recognition," IEEE Trans. Syst., Man, Cybern., vol. 13, pp. 826-834, Sept./Oct. 1993.

[8] R. C. Eberhard, R. W. Dobbins, and W. R. S. Webber, "CaseNet: A neural network tool for EEG wave form classification," in Proc. IEEE Symp. Computer-Based Medical Systems, 1989, pp. 60-89.

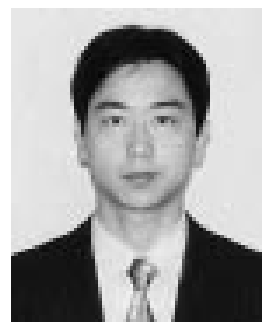

Takamasa Shimada completed doctoral course and received the Ph.D. degree in engineering from the University of Tsukuba Tsukuba, Japan, in 1996.

He was a Research Fellow of the Japan Society for the Promotion of Science from 1996 to 1998 . He is currently an Instructor at Applied Superconductivity Research Laboratory, Tokyo Denki University, Tokyo, Japan. In the field of medical electronics, his main research interests are EEG and MRI analysis and signal and image processing using neural networks and fuzzy rules.

Dr. Shimada is member of the Institute of Electronics, Information and Communication Engineers and the Japan Society of Medical Electronics and Biological Engineering. 


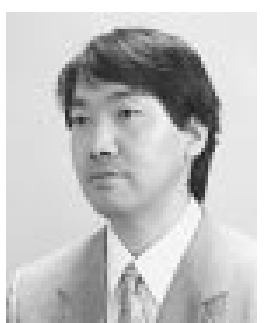

Tsuyoshi Shiina (M'87) graduated from the Electronic Engineering Department, the University of Tokyo, Tokyo, Japan, in 1982. and the M.S. and $\mathrm{Ph} . \mathrm{D}$. degrees in electronic engineering from the University of Tokyo, Tokyo, Japan, in 1984 and 1987, respectively.

From 1982 to 1987, he was with the Institute of Medical Electronics, the Faculty of Medicine, the University of Tokyo From 1987 to 1992, he was Lecturer and Associate Professor at the Department of Electronic Engineering, Tokyo University of Agriculture and Technology, Tokyo, Japan. In 1992, he moved to the University of Tsukuba, Tsukuba, Japan, as an Associate Professor of Institute of Information Sciences and Electronics. From 1996 to 1997, he was with the Institute of Cancer Research and Royal Marsden NHS Trust in the U.K. as a Visiting Professor and did research on ultrasonic elasticity imaging. His current research interests include visualization technique of structural and functional bio-information, for example, ultrasonic tissue characterization, 3-D blood flow measurement and bio-signal processing based on EEG and MEG

Dr. Shiina is a member of the Japan Society of Medical Electronics and Biological Engineering and the Japan Society of EEG and EMG. He is an Associate Editor of Institute of Electronics, Information and Communication Engineer Transactions, and General Secretary and Councilor of Japan Society of Ultrasonics in Medicine.

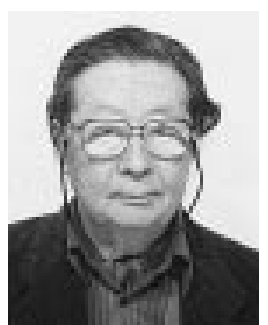

Yoichi Saito graduated from the Faculty of Medicine in 1952 and received the MD and DMSc. degrees in neuropsychiatry from the University of Tokyo, Tokyo, Japan. He continued his clinical and scientific work at the Department of Neuropsychiatry at the Hospital of the University of Tokyo.

From 1962 to 1963, he was with Harvard University, Cambridge, MA, as a Visiting Researcher. From 1966 to 1989 , he was an Instructor with the Faculty and the Graduate Course of Medicine, the University of Tokyo, and a Chief Staff at the Department of Neuropsychiatry in the University Hospital. In 1989, he retired the University Hospital and established the Research Institute for EEG Analysis with a neurology clinic. His research focuses on the quantitative diagnosis of psychiatric disorder, especially epilepsy, narcolepsy, and dementia, based on signal processing of EEG and evoked potentials.

Dr. Saito is a member of the Japan Society of Medical Electronics and Biological Engineering, Japan Society of EEG and EMG. 